\title{
De mares y ofrendas: la expresión del deseo lésbico en el cuento español tras la muerte de Franco
}

\author{
CONCEPCIÓN MARTÍN Huertas \\ Université Rennes 2
}

Resumen: Este artículo pretende analizar la evolución de la narrativa lesbiana desde la muerte de Franco hasta la actualidad a partir del estudio de tres cuentos: La búsqueda de Elizabeth (1982), de Marta Pessarrodona, Las virtudes peligrosas (1982), de Ana María Moix, y La semana más maravillosa de nuestras vidas (1997), de Cristina Peri Rossi. Con ellos abordaremos diversas problemáticas que van desde la búsqueda de la identidad hasta la indagación en el propio deseo y en un lenguaje que permita expresarlo.

Palabras clave: Cuento, lesbianismo, Marta Pessarrodona, Ana María Moix, Cristina Peri Rosi.

De recherches, de mers et de regards : l'expression du désir lesbien dans le conte espagnol après la mort de Franco

Résumé: Cet article propose d'analyser l'évolution du récit lesbien depuis la mort de Franco jusqu'à aujourd'hui à partir de l'étude de trois contes : La búsqueda de Elizabeth (1982), de Marta Pessarrodona, Las virtudes peligrosas (1982), d'Ana María Moix, et La semana más maravillosa de nuestras vidas (1997), de Cristina Peri Rossi. Ces oeuvres nous permettront d'aborder diverses problématiques, depuis la recherche de l'identité jusqu'à l'étude de son propre désir et d'un langage qui voudra bien l'exprimer.

Mots-clé: Conte, lesbianisme, Marta Pessarrodona, Ana María Moix, Cristina Peri Rosi. 
«A Chloe le gustaba Olivia...». No os asustéis. No os ruboricéis. Admitamos, en la intimidad de nuestra compañía, que a veces estas cosas ocurren. A veces a las mujeres les gustan las mujeres. «A Chloe le gustaba Olivia» - leí-. Y entonces caí en la cuenta del enorme cambio que ahí había. A Chloe le gustaba Olivia quizá por primera vez en la literatura.

Virginia Woolf, Un cuarto propio

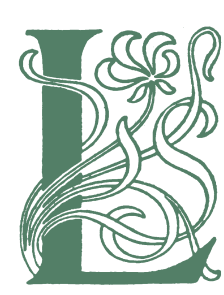

a literatura española, debido sobre todo a la Guerra Civil y al franquismo, tardó mucho más que las literaturas vecinas en decir "a Chloe le gustaba Olivia". De hecho, se podría decir que la narrativa no lo expresó explícitamente y con libertad hasta 1975, con el cuento de Carme Riera Te entrego, amor, la mar, como una ofrenda.

El objetivo de este estudio es hacer un breve análisis de la narrativa lesbiana española tras la muerte de Franco a partir de tres cuentos: La búsqueda de Elizabeth (1982), de Marta Pessarrodona, Las virtudes peligrosas (1982), de Ana María Moix, y La semana más maravillosa de nuestras vidas (1997), de Cristina Peri Rossi. La elección de los cuentos se debe tanto a una cuestión temática -queríamos mostrar los distintos modos de abordar las relaciones homoeróticas- como cronológica, ya que se busca hacer una comparativa entre la narrativa de los años ochenta y la de los noventa para mostrar su evolución.

Pero, antes de comenzar a hablar de la literatura lesbiana en España, cabría preguntarse qué se entiende por tal, ya que «tampoco existe, desde la teoría y crítica lesbiana una definición clara y unívoca de lo que es un texto lesbiano y cuáles deben ser sus contenidos o sus pautas formales, ni por asomo una versión viable y práctica de la categoría teórica literatura lesbiana» (Simonis, 2009: 50). A día de hoy, la crítica aún no ha sido capaz de acordar una definición única sobre lo que se debe entender por narrativa lesbiana. Mientras que para muchos solo es necesario que algún personaje femenino, protagonista o no, sienta atracción por otras mujeres, otros, más exigentes, precisan que la trama lésbica sea central. En cuanto a la autoría encontramos también discusiones acerca de si la autora debe ser homosexual o si una autora heterosexual o incluso un autor masculino también pueden hacer literatura lesbiana. 
Dado el desacuerdo, hemos optado por dar una definición propia. Temáticamente, entendemos que, para hablar plenamente de narrativa lesbiana, las relaciones entre mujeres tienen que ser uno de los temas centrales y no un episodio meramente anecdótico. Por ejemplo, no consideraríamos una muestra de literatura lesbiana Los soldados lloran de noche, de Ana María Matute que, en cambio, sí que lo es para autores como Wilfredo Hernández (2003). También creemos imprescindible que estas relaciones sean tratadas desde la dignidad, no patologizándolas, demonizándolas o haciendo escarnio de ellas, lo que fue común en la literatura hasta la muerte de Franco. En relación al autor, nuestra posición es más flexible que la de la mayoría de críticos, puesto que aunque los tres textos escogidos para este trabajo están escritos por mujeres, no creemos que esta narrativa sea exclusiva de un género y mucho menos de autoras lesbianas, como defienden algunos.

Según esta definición, los primeros textos que se podrían considerar propiamente lesbianos se editan en la Barcelona prácticamente inmediata a la muerte del dictador. Como afirma José Teruel, no es casual que se editasen allí, puesto que hubo un «pionero protagonismo de Cataluña en el movimiento de liberación gay en el tardofranquismo de los años setenta» (2013: 184). Gran importancia tiene el movimiento de liberación sexual barcelonés conocido como Gauche divine ya que, como indica María Castrejón «El ideario de la Gauche divine catalana rompe las estructuras vigentes, por lo que es posible introducir nuevos esquemas, tanto formales como temáticos; y es aquí donde nace la novela lésbica española» (2008: 41).

El primer texto lesbiano, como decíamos, se publica en 1975. Es el cuento Te deix, amor, la mar, com a penyora, de la mallorquina Carme Riera, el cual será traducido al castellano por la propia autora en 1980. Pero quizá el texto más importante, el que para muchos sienta las bases de la literatura lésbica en España, es El mismo mar de todos los veranos (1978), de Esther Tusquets.

Estas nuevas autoras se enfrentan a un gran reto literario: el de buscar un nuevo lenguaje para la creación de un discurso que carece de referentes previos. La expresión del deseo lésbico, su identificación o las descripciones del cuerpo femenino como sujeto y objeto de placer son solo algunos ejemplos de los desafíos que se empiezan a encarar en la literatura de la Transición. 
La reprivatización de la literatura tras la muerte de Franco y la necesidad de contar que experimentan los escritores -y especialmente las escritoras- se configuran como un escenario idóneo para el surgimiento de la literatura lesbiana. Estos primeros relatos están escritos casi siempre en primera persona, teniendo la memoria un papel primordial en ellos. Además se aprecia una búsqueda constante de interlocutor. Lo importante no es solo recordar, sino que alguien escuche nuestros recuerdos. Buena muestra de ello es la proliferación de la narración epistolar, como en Te entrego, amor, la mar, como una ofrenda, cuya autora va un paso más allá al escribir otro cuento/carta a modo de respuesta, Y pongo por testigo a las gaviotas (1977). La comunicación ya no es solo unilateral, sino que provoca una respuesta.

Una de las técnicas más utilizadas en la literatura lesbiana de estos años es el juego entre ausencia y presencia, entre lo dicho y no dicho o lo insinuado. El lector debe mantenerse alerta e ir descifrando las señales que le permitan entender. En el cuento de Riera se nos esconde el género del destinatario de la carta hasta las últimas líneas, si bien se nos da alguna pista que nos permite sospecharlo; en Las virtudes peligrosas, como tendremos tiempo de analizar más adelante, la relación entre las dos mujeres solamente se insinúa, pero nunca llega a consumarse de una manera carnal. Es bastante ilustrativo constatar la ausencia de la palabra "lesbiana" en estas narraciones. Pese a esto, no cabe duda es de que en esta época aparecen muchas escritoras que crean un nuevo modelo de mujer. Una mujer que pasa de ser objeto a ser sujeto de deseo, que va identificando y admitiendo y, sobre todo, eligiendo sus preferencias sexuales, y que se va despojando poco a poco de unos roles de género preestablecidos por una sociedad dominada por el patriarcado.

Podemos decir, como afirma Simonis, que «con un goteo lento pero constante, la escritura lesbiana va mostrándose cada vez más, conforme finaliza el siglo $\mathrm{xx}$, atreviéndose obra a obra a deshacerse de los velos que la tradición le impuso» (2009: 200). Esta escritura va asentándose, descubriendo por fin ese modo de expresión propio que se empezaba a esbozar en los ochenta. Donde más se advierte el cambio es en los temas. La literatura se va haciendo más explícitamente lesbiana. Las relaciones entre mujeres están cada vez más normalizadas y las protagonistas siguen un camino personal de «auto-reconocimiento» (Martín, 2002: 5).

Pero este auto-reconocimiento va más allá de la propia aceptación y busca también la aceptación del entorno. Frente a las primeras narraciones, en las 
que las relaciones entre mujeres se mantenían en el ámbito de lo privado, en las obras de los 90 se aprecia una mayor apertura social. Las protagonistas ya no se mantienen "en el armario" ante sus círculos más estrechos -familia, amigos cercanos- sino que ahora luchan por poder mostrarse también en otros ámbitos de índole pública. Por fin, y después de tanto tiempo, las relaciones homoeróticas comienzan a verse con naturalidad. Desaparecen los estereotipos y los tópicos, y las parejas homosexuales, como veremos en el cuento de Cristina Peri Rossi, siguen los mismos parámetros que las relaciones heterosexuales, tanto positiva como negativamente. Otro avance a partir de esta década es la expresión del goce femenino. El placer se expresa de una manera completamente explícita como culminación del proceso de búsqueda de un lenguaje propio.

Todos estos rasgos y esta evolución de la narrativa lesbiana se ven claramente en los tres cuentos que vamos a analizar a continuación. Comenzaremos con La búsqueda de Elizabeth, que es también la búsqueda de la identidad lesbiana; continuaremos con Las virtudes peligrosas y la imposibilidad de expresar el deseo fuera de los límites heteronormativos; y, finalmente, abordaremos La semana más maravillosa de nuestras vidas para romper con los estereotipos de una vez por todas.

\section{LA BÚSQUEDA DE ELIZABETH, DE MARTA PESSARRODONA: TRAS LOS PASOS DE LA IDENTIDAD}

La búsqueda de Elizabeth es un cuento de la catalana Marta Pessarrodona, que se publica en 1982 en la antología Doce cuentos de mujeres, coordinada por Ymelda Navajo. Es la historia de una búsqueda. La protagonista quiere encontrar a Elizabeth, si bien poco a poco vamos descubriendo que no se trata de un personaje de carne y hueso como cabría suponer, sino que lo que se busca es la propia identidad sexual. Como indica Gutiérrez-Rivas, «La identidad sexual de la protagonista se conocerá por medio del escrutinio de las distintas "pistas lingüísticas" tras las que se enmascara una sexualidad "no canónica"» (2012: 33). En este análisis iremos mostrando esas "pistas", así como el proceso de autorreconocimiento y aceptación que va experimentando el personaje principal. 
La ya citada Carolina Gutiérrez-Rivas, en su análisis de este cuento, establece una estructura, a nuestro modo de ver, muy acertada. Según ella, la historia, «claramente delimitada en tres etapas, plasma las diferentes fases de autoconocimiento y autoidentificación que atraviesa la protagonista» (2012: 35). Estas etapas son: Londres, $1976^{1}$, aparición y rechazo; Barcelona, 1979, reencuentro y conocimiento; y epílogo sin fecha, búsqueda y aceptación. La elección de Londres y Barcelona no es casual, puesto que Londres fue una capital en la que el feminismo estuvo muy activo en una época en la que en España no hablaba aún de la libertad sexual y Barcelona, como ya mencionamos en la introducción, fue la ciudad en la que aparecieron los primeros movimientos de liberación gay.

Es en la primera etapa, en Londres, donde la protagonista conoce a Elizabeth, es decir, donde empieza a descubrir en sí misma una identidad sexual "no canónica". La conoce tras una reunión «de un grupo que nunca se denomina "feminista" (pero se sobreentiende por el tipo de temas que se tocan durante la concentración: anticonceptivos y aborto)» (2012: 36). Elizabeth no llega a esa reunión, en la que iba a dar «un informe medio ensamblado sobre conocer el propio cuerpo» (Pessarrodona, 1982: 147) ${ }^{2}$. El tema del autoconocimiento, primer paso en el descubrimiento de la identidad sexual, no estará presente en la reunión pero sí acompañará a la protagonista a casa. Y es en este trayecto donde surge la afirmación que se convertirá en el leitmotiv del cuento: «Elizabeth me dijo que el cuerpo de una mujer es mucho más poético» (145). Una afirmación que en un principio la protagonista querrá rechazar. «Le dije, tal vez sin venir a cuento, de golpe, "soy heterosexual"» (148). $\mathrm{Si}$ en nuestro estudio introductorio veíamos que la narrativa lesbiana de los 80 estaba marcada por las tensiones entre lo dicho y lo no dicho, el hecho de que la protagonista diga, de forma tan abrupta y «sin venir a cuento», «soy heterosexual» debe hacer saltar las alarmas del lector avispado. Hay una negación de la identidad, un rechazo.

En esta primera etapa, se ve también como la protagonista tiene una relación con un hombre. Cuando Jordi, su novio, va a visitarla a Londres, ambos afirman estar «en el mejor momento de nuestras relaciones» (150), si bien en

1 En el trabajo de Gutiérrez Rivas se indica que la primera etapa es Londres, 1977 (2012: 35), si bien en el cuento de Marta Pessarrodona la fecha que se da es 1976 (1982: 145).

2 A partir de este momento, cuando se citen fragmentos del cuento, se indicará únicamente la página de la edición escogida en la que se encuentran. 
este tiempo ella ve «un par de veces más a Elizabeth», lo que se podría interpretar como la puesta en duda de su orientación plenamente heterosexual. En la figura de Jordi se ve también el pensamiento heteronormativo omnipresente en la España inmediatamente posterior al franquismo. Cuando su novia le cuenta lo que ha estado haciendo las últimas semanas, él le dice «iA ver si ahora te gustarán las mujeres!» (152), pero se lo dice «con la más rotunda tranquilidad, simplemente para hacerse el gracioso, sin el más mínimo tono de peligro, o de miedo» (152). No tiene miedo porque la posibilidad de que eso sea así, en su concepción heteronormativa y esencialista de la sexualidad, simplemente no existe.

La etapa en Barcelona comienza con el relato de la ruptura de la protagonista con Jordi y del comienzo de una nueva relación. En una primera lectura, al igual que pasa con el cuento de Riera, nuestro pensamiento heteronormativo nos hace dar por hecho que Toni, su nueva pareja, es un hombre, sin caer en la cuenta de que ese hipocorístico puede hacer referencia tanto a Antonio como a Antonia. Tras una lectura un poco más minuciosa, sin embargo, podemos encontrar algunas pistas lingüísticas que insinúan que se trata de una mujer. En primer lugar, y conociendo ya Te entrego, amor, la mar, como una ofrenda, habría que empezar a sospechar de la carencia de marcas gramaticales de género. Además, la narradora hace referencia constantemente a la novedad de su relación con respecto a todas las anteriores: «Una persona absolutamente nueva y diferente, que en nada se parecía a mi mundo habitual (un mundo del que nunca me había movido sentimentalmente), una persona con la que no se repetiría nada -ni errores un aciertos anteriores-, una persona única» (153). La relación con Toni es completamente distinta a las que se ven en el mundo habitual de la narradora -un mundo heteronormativo-, por lo que todo es nuevo para ella. Pero quizá la referencia más explícita al género de Toni tiene lugar cuando la narradora le dice: «Elizabeth tiene la culpa de que me gustes» (153). Por el final del capítulo puede suponerse que pese a la ilusión de liberación que le produce la relación con Toni en un primer momento, finalmente el miedo de la protagonista a ser sincera y a mostrarse tal y como es ante el resto del mundo provoca la ruptura amorosa.

En el comienzo del epílogo sin fecha, cuando la narradora ha empezado a olvidarse de Elizabeth, esta reaparece. Esta vez junto a «una chica de aspecto poco habitual, insólito en el barrio» que parece, por fin, empezar a abrirle los ojos. También se empieza a ver una relación más fluida entre la protagonista 
y Elizabeth -largas charlas, encuentros más casuales-, lo que culminará en la aceptación de la propia identidad. Aun así, y por última vez, nuestra protagonista interrumpe el contacto entre ellas: «Me pregunto si no interrumpí mi relación conmigo misma, si tanta complicación, una vez más, no me disparó fuera de la órbita» (157). En palabras de Gutiérrez-Rivas:

La decisión de interrumpir por un tiempo su relación con Elizabeth ofrece una vez más la evidencia inequívoca de lo que ya he dicho en líneas anteriores: Elizabeth es una parte de sí que $\mathrm{X}$ no logra resolver, que no logra asumir y con la que no quiere terminar de identificarse, por lo que una vez más decide apartarla de su vida $(2012,45)$.

Pero finalmente, la protagonista siente que debe hablar con Elizabeth, por lo que la busca tanto en Londres como en Barcelona sin éxito. Nadie la conoce, parece como si no hubiera existido nunca, lo que refuerza la idea de que se trata de la propia conciencia de la narradora y no un personaje real. La última afirmación, «Nadie puede orientarme con respecto a encontrar a Elizabeth» (159) puede interpretarse de distintas formas. Para Gutiérrez-Rivas, hace referencia al trabajo en solitario que cada homosexual debe realizar para admitirse a sí mismo como tal, «en el que únicamente intervienen el individuo y su conciencia» $(2012,45)$. En cambio, para nosotros la interpretación es otra, si bien ambas no son excluyentes. Consideramos que se trata más bien de una alusión a la heteronormatividad de la sociedad, puesto que en la época en la que transcurren los hechos -y en la que se escribe el cuentoes muy complicado encontrar a alguien que pudiera orientar a una mujer en su búsqueda de una expresión homoerótica de su propio deseo.

La búsqueda de Elizabeth es un cuento que muestra por primera vez en España el proceso de autoconocimiento y autoaceptación del sujeto lésbico en una sociedad que no facilita esta empresa. Asimismo, posee un final novedoso, podría decirse que adelantado a su época. Frente a los finales trágicos o frustrados de la mayor parte de la narrativa lesbiana de la Transición, Marta Pessarrodona nos abre una ventana a la esperanza con una mujer que se acepta a sí misma y que por fin deja de tener miedo y escapa a la convención social. La búsqueda de la protagonista tiene un objetivo: el de decirle a Elizabeth que sí, que lo sabe, que «el cuerpo de una mujer es mucho más poético», que por fin, y tras todo el proceso, ha sido capaz de sobreponerse a sus miedos y aceptarse. 
Aún quedan unos cuantos años para ver procesos de coming out completos y en los que no solo se consigue la aceptación personal, sino también la social, pero creemos que este primer paso en 1982 es crucial para ello.

\section{LAS VIRTUDES PELIGROSAS, DE ANA MARÍA MOIX, Y LOS LÍMITES DEL DESEO}

Las virtudes peligrosas aparece por primera vez en Doce relatos de mujeres (1982), si bien tres años más tarde su autora, Ana María Moix, lo incluirá en una antología de cuentos homónima. Todo el cuento gira en torno a la "misteriosa" relación de dos mujeres ya ancianas que, aunque nunca llegan a hablar ni a mantener contacto físico, tienen, durante su juventud, una conexión muy especial basada únicamente en sus miradas. Conexión que nadie consigue entender o descifrar y que incluso lleva a la locura al marido de una de ellas. El narrador, cuya identidad no se revelará hasta el final del relato, se dirige a Alice, la chica que va a leer a casa de ambas ancianas, supuestamente invidentes, para que esta intente comprender la historia de estas dos mujeres.

La información tanto de la identidad del narrador como de la historia de las dos ancianas se va suministrando de manera paulatina. En un principio, la naturaleza de la relación entre las dos mujeres no está clara. Parece tratarse de una simple relación vanidosa, de un vínculo superficial y egocéntrico consistente en el regocijo de la belleza contemplada de manera especular. Esta idea se refuerza con la imagen de los espejos cubiertos con velos de la anciana y, sobre todo, con el texto que esta le hace leer constantemente a Alice. Pero, según va avanzando la historia, algunos indicios nos hacen sospechar de que la relación va más allá.

De nuevo estamos ante un cuento en el que hay que descifrar las pistas lingüísticas que se esconden entre lo velado y lo revelado, entre lo dicho y lo sugerido. En primer lugar, debemos fijarnos en los adjetivos utilizados para describir las miradas de las dos mujeres. Estas aparecen como «penetrantes, hirientes pero melancólicas, resignadas pero expectantes» (Moix, 1982: 40)³, lo que parece hacer más referencia a una pasión amorosa que a una mera

\footnotetext{
V. Nota 4
} 
contemplación narcisista. La melancolía por lo que se desea pero no puede alcanzarse, la resignación ante esa imposibilidad, la expectación que surge como un brote de esperanza. Además abundan los adjetivos carnales, corporales. Las miradas son «penetrantes», hieren. Se habla de la «mirada hecha tacto» y de un «invisible abrazo» (46), lo que dota a este vínculo de un gran erotismo. Se pasa de la pura contemplación a una relación absolutamente sensorial.

Nancy Vosburg considera que una muestra indudable de que se trata de una relación lésbica reside en el hecho de que, cuando ambas son ancianas, sustituyen las miradas por el intercambio de objetos: «Their silent gestures, which have replaced the gaze in old age, suggest that this bond is something more than narcissistic recognition -that it is, indeed, a lesbian desire, that desire condemned, due to social, cultural, and personal limitations» (2003: 83).

La condena social y cultural a la que hace referencia Vosburg también se puede identificar en otros lugares del texto. Durante la representación operística, el general, marido de una de las mujeres, espera con ansiedad el momento en el que se enciendan las luces y en el que «el despertar de la vida pública y social» (47) destruya el vínculo creado entre ellas. Pero eso no va a ocurrir, puesto que ambas abandonan su palco antes de que se acabe la función, desafiando de este modo la presión social. La mirada femenina ejerce el poder que no pueden ejercer sus palabras, y este poder vuelve irrelevante a la mirada masculina. En cambio, las protagonistas no hablan porque el lenguaje respalda ni puede expresar lo que sienten, puesto que está en manos del sistema heteropatriarcal. Nancy Vosburg está en contra de esta teoría:

As I read others who have read moix, however, my pleasure momentarily wanes. Their readings emphasize women's problematic relationship to language; the ultimate power of the male gaze, because the narrative control is patriarcal; the entrapment of women's sexuality in male-dominated discourse; the lack of viability of the unnamable desire given its spatial and discursive marginality. Their readings suggest that I have been taken in by the artífice and point to my own complicity in the silencing woman (2003: 85).

Para ella, esta interpretación del silencio de las mujeres debido a la dominación masculina del lenguaje supone la complicidad del lector con esta con- 
vención literaria. En cambio, desde nuestro modo de entender este cuento, el lector juega un papel completamente opuesto. Para empezar, consideramos innegable que se trata de un texto que expresa tanto la imposibilidad del lenguaje ante las relaciones homoeróticas, como la dominación heteropatriarcal del discurso. Las dos mujeres intercambian miradas porque mediante las palabras no pueden expresar libremente sus sentimientos, en primer lugar, porque la sociedad no se lo permitiría y, en segundo lugar, porque el propio lenguaje carece de modelos para ello. Este silencio se extiende también al general, que experimenta una gran impotencia al no poder expresar sus sospechas y sus celos libremente, puesto que no encuentra palabras para definir la relación de su mujer con la desconocida: «no poder referirse a ellos [los celos] sin nombrarlos dada su naturaleza incorpórea, fantasmal» (50). Además, pese a que se trata de una historia de amor lesbiano, las mujeres no tienen voz -ni siquiera a Alice, a la que se dirige el texto, se le permite responder-, y el narrador, paradójicamente, es un hombre. Que sea una voz masculina la que narra la relación entre dos mujeres es la mejor muestra de que el texto quiere poner de manifiesto la dominación masculina del discurso.

En cuanto a la complicidad de la que habla Vosburg, sí que creemos que se establece una complicidad con el lector, pero esta consiste en el desciframiento de la verdadera naturaleza de la relación entre las dos mujeres, en la "lectura" de su silencio, y no en la perpetuación de los modelos heteropatriarcales. Al igual que en el cuento anterior nadie podía ayudar a la protagonista a encontrar a Elizabeth porque para la sociedad heteronormativa ella parecía no existir, en Las virtudes peligrosas la relación entre las dos mujeres se convierte en un misterio que nadie llega a entender. La frase «el general no comprendió» (42) actúa como leitmotiv que culmina con la pregunta del narrador « ¿Comprendes, ahora, Alice?» (64). Alice es la lectora y a la vez es el vínculo entre las dos mujeres. Rudolph le pide que vuelva a recordar la historia, puesto que en su subconsciente está la clave para "entender". Estamos, pues, ante un texto que insta al lector a ahondar en su interior y a volver a mirar para lograr comprender.

Alice, además de con el lector, puede asimilarse también con las nuevas generaciones, debido a su corta edad, por lo que quizá simbolice también una mirada optimista hacia el futuro. Ella es la clave para que Rudolph, quien hasta ese momento no había logrado entender, por fin entienda. 
Las virtudes peligrosas es, en definitiva, una historia que señala las dificultades del normal desarrollo de las relaciones homoeróticas en una sociedad que las rechaza, mostrando un deseo femenino reprimido e irrealizable. Es un cuento que pone sobre la mesa la problemática de la imposibilidad del lenguaje y que, además, busca una mayor aceptación y comprensión de las relaciones lesbianas.

\section{LA SEMANA MÁS MARAVILLOSA DE NUESTRAS VIDAS, CRISTINA PERI ROSSI}

La semana más maravillosa de nuestras vidas, de Cristina Peri Rossi, se publica ya bien avanzada la década de los noventa, exactamente en 1997. En este cuento, la narradora conoce a una mujer en un bar de ambiente y se van juntas a un hotel. A los dos días, esta descubre que su amante está casada con un hombre, lo que le genera incomodidad y dudas que se acrecentarán cuando conozca al marido.

En lo que respecta a la expresión del lesbianismo, estamos ante un texto completamente distinto a los dos anteriores. Ya no encontramos las dificultades que había en los ochenta para expresar el deseo lesbiano mediante el lenguaje. Aquí a cada cosa se le llama por su nombre y, cuando se usan eufemismos o imágenes distorsionadas, es para aportar un tono irónico. Además, las protagonistas ya no luchan por entenderse o por aceptarse y tampoco se esconden, si bien sus relaciones tampoco son perfectas. Hay un igualamiento de las relaciones homosexuales y heterosexuales, los problemas de ambas son los mismos porque el género de los amantes no influye en la esencia de la relación:

Cristina Peri Rossi no defiende de forma maniquea el amor lesbiano, defiende el amor total y libre que lucha por existir, que supera las complicaciones, que no el más maravilloso de nuestras vidas, pero «es», sin importar el género de los amantes sino su capacidad de amar, de fundirse en el otro sin necesidad de perder la identidad (Castrejón, 2008: 89).

Así vemos, por ejemplo, que cuando la protagonista prevé que va a discutir con su amante y reflexiona sobre la necesidad de las discusiones en las parejas nos dice que «Ocurre con las homosexuales y con las heterosexuales» (Peri, 2007: 640) ${ }^{4}$.

$4 \quad$ V. Nota 4. 
La semana más maravillosa de nuestras vidas es una constante ruptura de tópicos relacionados con el amor, con las relaciones, con el sistema patriarcal y con el matrimonio. Para ello, Peri Rossi hace uso de la ironía, lo cual también supone una novedad. Hasta mediados de los noventa, los autores de literatura lesbiana no empezarán a incluir el humor, la ironía o el sarcasmo en sus obras, sino que estas serán, como ya hemos visto, de corte más serio e introspectivo.

La desmitificación del amor romántico es más que evidente. No hay amor en el matrimonio -institución canónica del amor romántico- sino rutina y formalismos. El verdadero amor, en este cuento, está en las relaciones extramatrimoniales, en las amantes que se conocen en un bar y en el sexo esporádico y desenfrenado en una suite de hotel. Así, se nos dice «¿No sabes que tres años, tres meses y tres días es el tiempo justo que dura la pasión? Todo lo demás -continué con ironía -es matrimonio» (640). Además, los gestos que normalmente se consideran románticos, aquí carecen de valor, como el ramo de flores que Frank regala a su mujer y que acaba tirado debajo de la cama. Y, además de desvincular completamente el amor y el matrimonio, este se relaciona con la rutina, con la falsedad y las apariencias e incluso con el dinero y la conveniencia. Así, la narradora afirma: «las mujeres casadas no me gustan -continué-. Llevan un anillo al dedo, siempre están insatisfechas y mezclan el amor con el dinero» (638). También se llega a decir de esta institución que es un «manicomio» o una «fábrica de locos» (645).

En el uso del lenguaje, como ya hemos dicho, las diferencias entre este cuento y los dos anteriores son bastante notables. La ruptura de tópicos no solo es temática sino también lingüística. Así, por ejemplo, el tópico del amor disfrazado de amistad que veíamos en los 80 se reinventa aquí con un significado nuevo. Si para Riera las relaciones entre mujeres no se podían nombrar y por ello se disfrazaban de amistad: «Te obsesionaba la idea de que yo algún día pudiera reprocharte aquel amor que llamábamos amistad» (1980: 14), para Peri Rossi cuanto menos marcado está algo por el lenguaje, más auténtico es: «El hecho de que no exista una palabra para nombrar esta clase de relación es la prueba de su autenticidad. Lucía y yo somos amigas» (639).

El tópico de la ausencia de palabras y de la imposibilidad del lenguaje ante las relaciones homoeróticas también es reutilizado por la autora. Ya no hay tabúes para relatar los encuentros sexuales entre mujeres, ahora el problema 
está en la insuficiencia de las palabras en los discursos amorosos, independientemente del género de los amantes:

No hablamos de ninguna de las cosas que teníamos pendientes. Los diálogos de las personas enamoradas, si fueran grabados, resultarían verdaderamente estúpidos. En cambio, si se filmaran las miradas, estas revelarían el goce. Un goce para el que no hay palabras, más que las triviales: «Me gusta tu pelo», «Chúpame», «Tócame», «Bébeme», «Abrázame», «Me gusta tu vientre» y cosas así (649).

Este goce de las miradas nos recuerda irremediablemente al cuento de Moix, si bien aquí los códigos cambian. Si las protagonistas de Las virtudes peligrosas no podían hablar porque el discurso no les pertenecía y, además, no tenían apoyo social, las del cuento de Peri Rossi, aunque ya son dueñas de sus propias palabras, consideran que estas no alcanzan para expresar su pasión.

También en relación con el cuento de Ana María Moix, encontramos otra inversión de los tópicos, en este caso de los referentes a la comprensión del lesbianismo. En Las virtudes peligrosas, el objetivo de Rudolph era que Alice "entendiese" las relaciones homoeróticas, puesto que estas resultaban incomprensibles para la sociedad del momento y, especialmente, para el marido de la anciana. En cambio, en el cuento de Peri Rossi, el marido de Eva conoce, entiende y respeta las relaciones lésbicas de su mujer, mientras que la narradora no entiende las relaciones matrimoniales canónicas.

El cuento de Peri Rossi muestra, pues, el consustancial cambio que experimenta la narrativa lesbiana en los noventa, sobre todo si lo confrontamos con los dos cuentos analizados anteriormente. Ya no se trata de una búsqueda de la propia identidad sexual, puesto que esta ya ha quedado definida y es aceptada con naturalidad por las protagonistas. No se ponen trabas al deseo, por lo que tampoco se trata ya de una historia de pasión frustrada y de imposibilidad. De lo que se trata es de romper con los tópicos, de cuestionar todos los estereotipos, de darles la vuelta o de reinventarlos. De universalizar el amor e independizarlo del género.

Como hemos visto a lo largo de este estudio, con el fin de la dictadura, algunas voces valientes se atrevieron a dar visibilidad, mediante sus textos, a algo que se había mantenido oculto y reprimido demasiados años, sin tener 
modelos previos que seguir y buscando, ante todo, la aceptación social. Gracias a estas voces, los escritores de los noventa tuvieron por fin unas bases sobre las que construir una narrativa moderna, comprometida y crítica que mostrase, desde la más absoluta naturalidad, el amor entre mujeres.

Actualmente, la narrativa lesbiana tiene una mayor presencia en el panorama literario español. Ya no hay miedo ni limitaciones a la hora de expresar el deseo homoerótico. Además, cada vez hay más editoriales que apuestan por esta literatura o incluso que publican exclusivamente textos con esta temática y, desde hace unos diez años, ya no es tan difícil encontrar estudios sobre literatura lesbiana. Aun así, aún queda mucho trabajo por hacer. Siendo necesario que la literatura lesbiana salga de las editoriales especializadas y tenga una mayor visibilidad en el mercado editorial. Para ello, las grandes editoriales deberían apostar por este tipo de historias y no discriminarlas y, al mismo tiempo, estas pequeñas editoriales especializadas no deberían publicar cualquier cosa, independientemente de su valor literario, simplemente porque la trama principal esté salpicada por relaciones homoeróticas.

También es fundamental la entrada de esta literatura en los circuitos académicos. Aunque cada vez es más habitual que aparezcan estudios sobre el lesbianismo en la literatura, todavía es bastante común que estos vengan de investigadores que trabajan en universidades extranjeras, especialmente estadounidenses.

Es indudable que la literatura lesbiana lleva ya un largo e importantísimo camino recorrido, que será fundamental para sentar las bases del camino que aún queda por recorrer. Un camino que seguro estará lleno de hombres y mujeres valientes y orgullosos de sus madres y de sus abuelas literarias, de Riera, de Tusquets, de Moix y de muchas más que se atrevieron a alzar la voz por primera vez para decir «a Chloe le gustaba Olivia».

\section{REFERENCIAS BIBLIOGRÁFICAS}

CAstrejón, María (2008), ...Que me estoy muriendo de agua: Guía de narrativa lésbica española, Egales, Barcelona. 
GutiéRrez-Rivas, Carolina (2012), «Pistas lingüísticas e identidad sexual en La búsqueda de Elizabeth, de Marta Pessarrodona», en Leonardo García Jaramillo (coord.), Co-herencia, Universidad EAFIT, Medellín, vol. 9, n. 17, pp. 31-49.

HERNÁNDEZ, Wilfredo (2003), «From the margins to the mainstream: lesbian characters in spanish fiction (1964-1979)», en Lourdes Torres e Inmaculada Pertusa (eds.), Tortilleras: Hispanics and U.S. latina lesbian expression, Temple University Press, Philadelphia, pp. 19-34.

Martín Armas, Dolores (2002), «Literatura española lesbiana de los noventa: Tres modelos», en Céfiro. Enlace hispano cultural, Texas Tech University, n. 2, pp. 4-8.

Morx, Ana María (1982), «Las virtudes peligrosas», en Ymelda Navajo (ed.), Doce relatos de mujeres, Alianza, Madrid, pp. 35-64.

Palma Borrego, Ma José (2005), «Literatura lesbiana española: un lugar casi desierto», en Patrick Dubuis y Nicolas Balutet (coord.), Littératures, arts, homosexualités: dossier bilingue de littératures hispaniques, Inverses, n. 5, pp. 202-209.

Peri Rossi, Cristina (1997), «La semana más maravillosa de nuestras vidas», en Cuentos reunidos, Lumen, 2007, pp. 636-657.

Pertusa, Inmaculada (2005), La salida del armario: lecturas desde la otra acera: Esther Tusquets, Carme Riera, Sylvia Molloy, Cristina Peri Rossi, Libros del Pexe, Gijón.

Pessarrodona, Marta (1982), «La búsqueda de Elizabeth», en Ymelda Navajo (ed.), Doce relatos de mujeres, Alianza, Madrid, pp. 143-159.

RIERA, Carme (1980), «Te entrego, amor, la mar, como una ofrenda», en Palabra de mujer, Laia, Barcelona, pp. 9-32.

Simonis, Angie (2009), Yo no soy ésa que tú te imaginas: El lesbianismo en la narrativa española a través de sus estereotipos, Centro de estudios sobre la mujer, Universidad de Alicante. 
Teruel Benavente, José (2013), «Representación del lesbianismo en la narrativa de la transición democrática», en María Jesús Zamora Calvo (ed.), La mujer ante el espejo: Estudios corporales, Abada, Madrid, pp. 183-201.

Vosburg, Nancy (2003), «Silent pleasures and the pleasure of silence: Ana María Moix's Las virtudes peligrosas», en Lourdes Torres e Inmaculada Pertusa (eds.), Tortilleras: Hispanics and U.S. latina lesbian expression, Temple University Press, Philadelphia, pp. 81-90.

- (2008), «Los ritmos eróticos del cuerpo literario lésbico: Riera, Tusquets, Moix y Company», en Beatriz Ferrús y Nuria Calafell (eds.), Escribir con el cuerpo, UOC, Barcelona, pp. 137-143. 


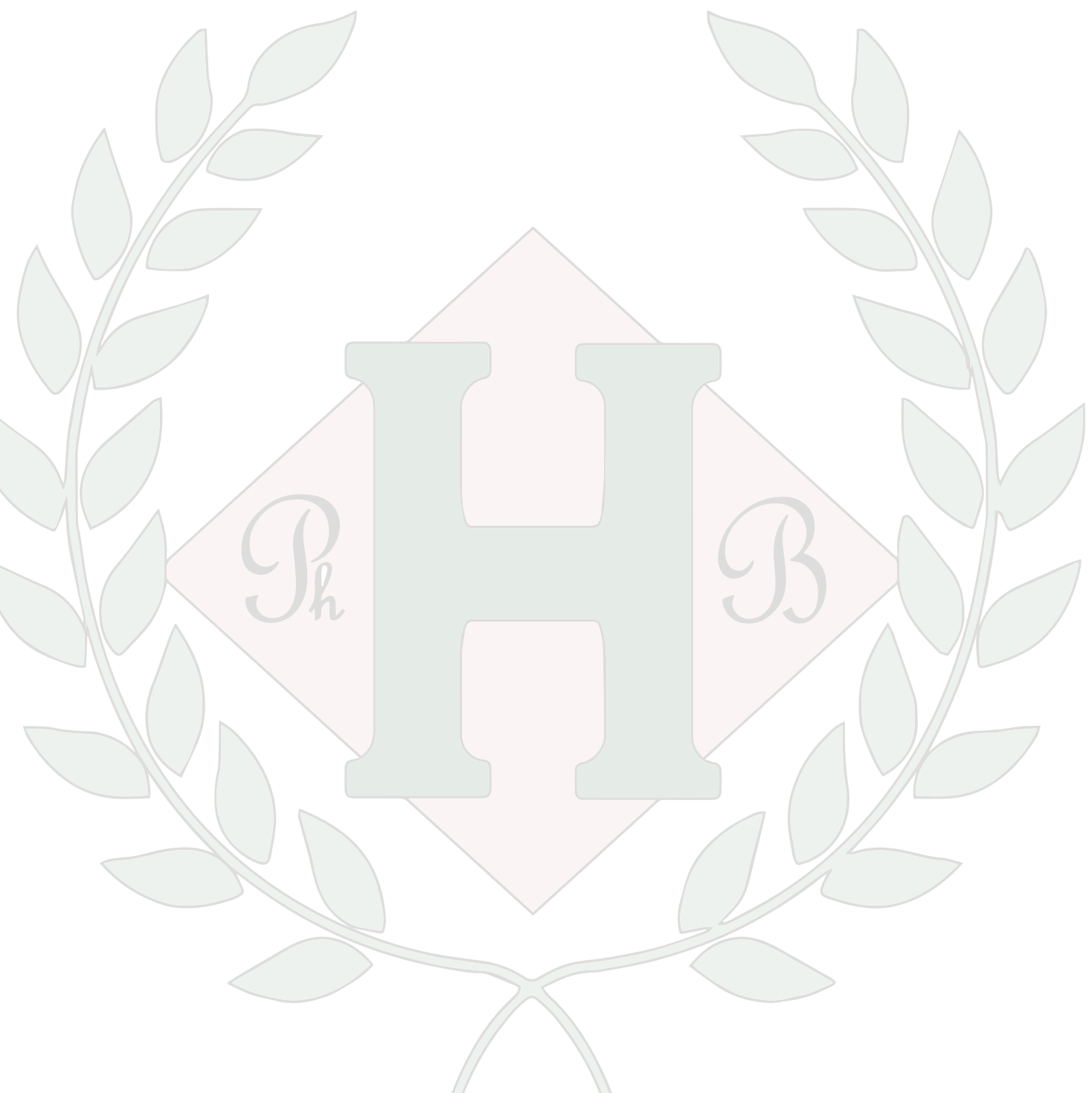

